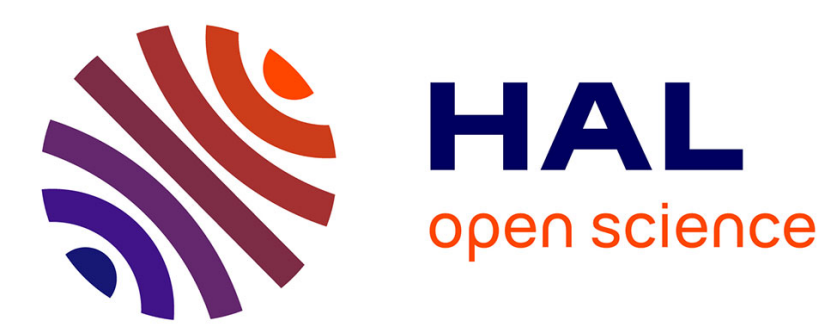

\title{
A Convex Semi-Definite Positive Framework for DTI Estimation and Regularization
}

\author{
Gilles Fleury, Radhouène Neji, Noura Azzabou, Nikolaos Paragios
}

\section{To cite this version:}

Gilles Fleury, Radhouène Neji, Noura Azzabou, Nikolaos Paragios. A Convex Semi-Definite Positive Framework for DTI Estimation and Regularization. International Symposium on Visual Computing, Nov 2007, Lake Tahoe, United States. pp. 220-229. hal-00258945

\section{HAL Id: hal-00258945 \\ https://hal-centralesupelec.archives-ouvertes.fr/hal-00258945}

Submitted on 26 Feb 2008

HAL is a multi-disciplinary open access archive for the deposit and dissemination of scientific research documents, whether they are published or not. The documents may come from teaching and research institutions in France or abroad, or from public or private research centers.
L'archive ouverte pluridisciplinaire HAL, est destinée au dépôt et à la diffusion de documents scientifiques de niveau recherche, publiés ou non, émanant des établissements d'enseignement et de recherche français ou étrangers, des laboratoires publics ou privés. 


\title{
A Convex Semi-Definite Positive Framework for DTI Estimation and Regularization
}

\author{
Radhouène Neji ${ }^{1,2}$, Noura Azzabou ${ }^{1}$, Nikos Paragios ${ }^{1}$, and Gilles Fleury ${ }^{2}$ \\ 1 GALEN Group, Laboratoire des Mathématiques Appliquées aux Systèmes, Ecole \\ Centrale Paris, Châtenay-Malabry, France \\ 2 Département Signaux et Systèmes Electroniques, Ecole Supérieure d'Electricité, \\ Gif-sur-Yvette, France *
}

\begin{abstract}
In this paper we introduce a novel variational method for joint estimation and regularization of diffusion tensor fields from noisy raw data. To this end, we use the classic quadratic data fidelity term derived from the Stejskal-Tanner equation with a new smoothness term leading to a convex objective function. The regularization term is based on the assumption that the signal can be reconstructed using a weighted average of observations on a local neighborhood. The weights measure the similarity between tensors and are computed directly from the diffusion images. We preserve the positive semi-definiteness constraint using a projected gradient descent. Experimental validation and comparisons with a similar method using synthetic data with known noise model, as well as classification of tensors towards understanding the anatomy of human skeletal muscle demonstrate the potential of our method.
\end{abstract}

\section{Introduction}

Diffusion tensor imaging (DTI) is an emerging non-invasive modality allowing the quantitative investigation of water protons diffusion within biologic tissues. Since diffusion is sensitive to the presence of organized structures, DTI is used mostly in brain studies and has become a tool to infer white matter connectivity [1]. Such a modality offers measurements of the amount of diffusion of water molecules in several different directions. One then can infer the estimation of a tensor which is a $3 \times 3$ symmetric positive definite matrix representing the uncertainty on the position of water protons with a Gaussian model of displacement.

However, the DTI experimental protocol yields noisy observations due to the diffusion-sensitizing magnetic gradient. Furthermore, the clinical protocols refer to relatively low magnet strength, or a rather low signal-to-noise ratio. Therefore, signal reconstruction is crucial to obtain an appropriate estimate of the tensor field and for subsequent use of this estimate in applications like fiber tractography.

Several methods have been proposed to address diffusion tensor regularization. In [2], a two-step regularization was proposed consisting of the restoration

* This work was partially supported by Association Française contre les Myopathies (AFM: http://www.afm-france.org) 
of the principal diffusion directions using a total variation-model followed by the smoothing of the eigenvalues using an anisotropic tensor-driven formulation. In [3], the maximization of a log-posterior probability based on the Rician noise model is considered to smooth directly the diffusion-weighted images. A Bayesian model based on a Gaussian Markov Random Field was used in [4] to smooth the diffusion tensors. In [5], the authors consider the tensors as lying on a Riemannian manifold and use the corresponding distance to derive a local weighted averaging for DTI denoising. Tensors are assumed to be positive-definite matrices which was taken into account in [6] where an anisotropic filtering of the $L^{2}$ norm of the gradient of the diffusion tensor was considered and their proposed PDE scheme constrains the estimation to lie on this space. Such a concept was further developed in [7] where a variational method was proposed that aimed to minimize the $L^{p}$ norm of the spatial gradient of the diffusion tensor under a constraint involving the non-linear form of Stejskal-Tanner equation. A non linear diffusion scheme is described in [8] where smoothing is made directiondependent using a diffusion matrix in the PDE system. More recently, in [9] a joint reconstruction and regularization was proposed in the context of an energy minimization in a Log-Euclidean framework. The existing variational methods focused disproportionately on enforcing the positive-definiteness constraint, with the regularization term usually chosen as a function of the norm of the gradient. The main limitation of most of the above-mentioned methods is the nature of the cost function (non-convex) that entails a preliminary initialization step, while little attention was paid to defining appropriate smoothness components that account for the expected nature of tensors.

In this paper we propose a new variational approach to jointly estimate and regularize diffusion tensor images. We use a convex energy functional which combines the linearized form of Stejskal-Tanner equation as a data fidelity term and a new regularization term involving precalculated weights which measure the similarity between neighboring tensors. We show the results of our method both on synthetic datasets and real data of diffusion tensor muscle images.

\section{DTI Estimation and Regularization}

Let us assume that $n$ DTI acquisitions $\left(S_{k}\right)_{k=1 \ldots n}$ with respect to different magnetic gradient directions $\left(\mathbf{g}_{k}\right)_{k=1 \ldots n}$ are available. Ideally, the expected signal at a voxel $\mathbf{x}$ for the direction $k$ as explained in [10] should respect the following condition

$$
S_{k}(\mathbf{x})=S_{0}(\mathbf{x}) \exp \left(-b \mathbf{g}_{k}^{t} \mathbf{D}(\mathbf{x}) \mathbf{g}_{k}\right)
$$

with the tensor $\mathbf{D}$ being the unknown variable and $b$ a value that depends on the acquisition settings. The estimation of the tensors in the volume domain $\Omega$ can be done through direct inference (6 acquisitions are at least available), which is equivalent to minimizing:

$$
E_{d a t a}(\mathbf{D})=\int_{\Omega} \sum_{k=1}^{n}\left(\log \left(S_{k}(\mathbf{x}) / S_{0}(\mathbf{x})\right)+b \mathbf{g}_{k}^{t} \mathbf{D}(\mathbf{x}) \mathbf{g}_{k}\right)^{2} d \mathbf{x}
$$


This energy is based on the linearized diffusion tensor model which is reasonable for moderate values of SNR [11]. Such a direct estimation is quite sensitive to noise, on the other hand, it refers to a convex term, which is rather convenient when seeking its lowest potential. The most common approach to account for noise is through the use of an additional regularization term which constrains the estimation of $\mathbf{D}$ to be locally smooth. One of the most prominent uses of DTI is fiber extraction. Therefore it is natural to assume that locally these fibers do have similar orientations. In such a context, the tensor can be expressed as a linear combination of the tensors lying in its neighborhood since they are likely to represent the same population of fibers. Such a regularization constraint was introduced in the case of image restoration in [12]. This assumption still holds at the boundaries between different groups of fibers as long as the linear combination is thoroughly chosen to ensure that the contribution of tensors belonging to a different fiber population is negligible. It is also more accurate than the underlying assumption of total-variation based approaches where the tensor field is considered piecewise constant. This leads us to define the following regularization component:

$$
E_{\text {smooth }}(\mathbf{D})=\int_{\Omega}\left\|\mathbf{D}(\mathbf{x})-\frac{1}{Z(\mathbf{x})} \int_{\mathbf{y} \in \mathcal{N}_{\mathbf{x}}} w(\mathbf{x}, \mathbf{y}) \mathbf{D}(\mathbf{y}) d \mathbf{y}\right\|_{F}^{2} d \mathbf{x}
$$

where $w(\mathbf{x}, \mathbf{y})$ reflects the similarity between tensors $\mathbf{D}(\mathbf{x})$ and $\mathbf{D}(\mathbf{y}),\|A\|_{F}$ being the Frobenius norm $\|A\|_{F}=\sqrt{\operatorname{tr}\left(A^{t} A\right)}$ and $Z(\mathbf{x})$ is a normalization factor, i.e $Z(\mathbf{x})=\int_{\mathbf{y} \in \mathcal{N}_{\mathbf{x}}} w(\mathbf{x}, \mathbf{y}) d \mathbf{y}$. The most critical aspect of such an approximation model is the definition of weights, measuring the similarity between tensors within the local neighborhood. The use of Gaussian weights is a common weight's selection, i.e $\left[w(\mathbf{x}, \mathbf{y})=e^{\frac{-d^{2}(\mathbf{D}(\mathbf{x}), \mathbf{D}(\mathbf{y}))}{2 \sigma^{2}}}\right]$, where $d(. ;$.$) is a distance between ten-$ sors and $\sigma$ a scale factor. In the context of direct estimation and regularization it is more appropriate to define similarities directly on the observation space rather than the estimation space. Such a choice will lead to a tractable estimation, while preserving the convexity of the cost function. Our distance definition as well as our minimization step are based on the representation of symmetric positive semi-definite matrices $S_{+}^{3}$ as a convex closed cone in the Hilbert space of symmetric matrices $S^{3}$, where the standard scalar product is defined by $\langle A, B\rangle_{F}=\operatorname{tr}\left(A^{t} B\right)$ which induces the corresponding Frobenius norm.

\subsection{Measuring Similarities from diffusion weighted images}

We aim at simultaneously estimating and smoothing the tensor field, therefore the weights $w(\mathbf{x}, \mathbf{y})$ in $E_{\text {smooth }}$ should be precalculated using the raw data. The most straightforward estimation of the distances can be done through the algebraic distance between the $\log \left(S_{k} / S_{0}\right)$ for two neighborhood voxels in any direction

$$
d(\mathbf{D}(\mathbf{x}), \mathbf{D}(\mathbf{y}))=\frac{1}{b} \sqrt{\sum_{k=1}^{N}\left(\log \left(S_{k}(\mathbf{x}) / S_{0}(\mathbf{x})\right)-\log \left(S_{k}(\mathbf{y}) / S_{0}(\mathbf{y})\right)\right)^{2}}
$$


One can easily show that such an expression does not reflect similarity between tensors according to the norm $\|.\|_{F}$. In fact, this leads to

$d(\mathbf{D}(\mathbf{x}), \mathbf{D}(\mathbf{y}))=\sqrt{\sum_{k=1}^{N}\left(\mathbf{g}_{k}^{t}(\mathbf{D}(\mathbf{x})-\mathbf{D}(\mathbf{y})) \mathbf{g}_{k}\right)^{2}}=\sqrt{\sum_{k=1}^{N}<\mathbf{D}(\mathbf{x})-\mathbf{D}(\mathbf{y}), \mathbf{G}_{k}>_{F}^{2}}$

where $\mathbf{G}_{k}=\mathbf{g}_{k} \mathbf{g}_{k}^{t}$ do not form necessarily an orthonormal basis. We use a Gram-Schmidt orthogonalization scheme to calculate an orthonormal basis $\widetilde{\mathbf{G}_{k}}$ such that $\widetilde{\mathbf{G}_{k}}=\sum_{l} \alpha_{k l} \mathbf{G}_{l}$ (each new vector of the new basis is a linear combination of the vectors of the initial basis). This procedure allows us to have an approximation of $\|\mathbf{D}(\mathbf{x})-\mathbf{D}(\mathbf{y})\|_{F}$ directly from the raw data $S_{k}$ and $S_{0}$ as follows

$$
\begin{aligned}
\|\mathbf{D}(\mathbf{x})-\mathbf{D}(\mathbf{y})\|_{F} & =\sqrt{\sum_{k=1}^{N}<\mathbf{D}(\mathbf{x})-\mathbf{D}(\mathbf{y}), \widetilde{\mathbf{G}_{k}}>_{F}^{2}} \\
& =\frac{1}{b} \sqrt{\sum_{k=1}^{N}\left(\sum_{l} \alpha_{k l}\left(\log \left(S_{k}(\mathbf{x}) / S_{0}(\mathbf{x})\right)-\log \left(S_{k}(\mathbf{y}) / S_{0}(\mathbf{y})\right)\right)\right)^{2}}
\end{aligned}
$$

\subsection{Semi-Definite Positive Gradient Descent}

One now can seek the lowest potential of the cost function towards recovering the optimal solution on the tensor space. Unlike the Riemannian approaches where non convex functionals are minimized [6], the present framework consists of a convex energy with a unique minimum which can be reached using a projected gradient descent on the space of semi-definitive positive matrices. The projection from $S^{3}$ onto $S_{+}^{3}$ denoted by $\Pi_{S_{+}^{3}}$ is well defined and has an explicit expression. Indeed, projecting $M$ amounts to replacing the negative eigenvalues in its spectral decomposition by $0[6,13]$. Note that we minimize over the set of semi-definite positive matrices because it is topologically closed, as opposed to the set of definite positive matrices. In the current setting, the problem is well posed and the projected gradient descent algorithm is convergent for a suitable choice of the time step $d t$. Using a weighting factor $\lambda$ between the data attachment term and the regularization energy, the gradient descent can be expressed as the following equation

$$
\begin{aligned}
\mathbf{D}^{t+1}(\mathbf{x}) & =\Pi_{S_{+}^{3}}\left(\mathbf{D}^{t}(\mathbf{x})-d t \frac{\partial E}{\partial \mathbf{D}(\mathbf{x})}\left(\mathbf{D}^{t}\right)\right) \\
& =\Pi_{S_{+}^{3}}\left(\mathbf{D}^{t}(\mathbf{x})-d t \lambda \frac{\partial E_{\text {smooth }}}{\partial \mathbf{D}(\mathbf{x})}\left(\mathbf{D}^{t}\right)-d t \frac{\partial E_{\text {data }}}{\partial \mathbf{D}(\mathbf{x})}\left(\mathbf{D}^{t}\right)\right)
\end{aligned}
$$


where

$$
\begin{aligned}
\frac{\partial E_{\text {smooth }}}{\partial \mathbf{D}(\mathbf{x})}(\mathbf{D})= & 2 \mathbf{D}(\mathbf{x})-2 \int_{\mathbf{y} \in \mathcal{N}_{\mathbf{x}}} \frac{w(\mathbf{x}, \mathbf{y})}{Z(\mathbf{x})} \mathbf{D}(\mathbf{y}) d \mathbf{y} \\
& -2 \int_{\mathbf{y} \in \mathcal{N}_{\mathbf{x}}} \frac{w(\mathbf{x}, \mathbf{y})}{Z(\mathbf{y})}\left(\mathbf{D}(\mathbf{y})-\int_{\mathbf{z} \in \mathcal{N}_{\mathbf{y}}} \frac{w(\mathbf{z}, \mathbf{y})}{Z(\mathbf{y})} \mathbf{D}(\mathbf{z}) d \mathbf{z}\right) d \mathbf{y} \\
\frac{\partial E_{\text {data }}}{\partial \mathbf{D}(\mathbf{x})}(\mathbf{D})= & 2 b \sum_{k=1}^{N}\left(\log \left(S_{k}(\mathbf{x}) / S_{0}(\mathbf{x})\right)+b \mathbf{g}_{k}^{t} \mathbf{D}(\mathbf{x}) \mathbf{g}_{k}\right) \mathbf{G}_{k}
\end{aligned}
$$

Let us define the norm $\|\cdot\|_{T F}$ over the whole tensor field $\mathbf{D}$ as $\|\mathbf{D}\|_{T F}=$ $\int_{\Omega}\|\mathbf{D}(\mathbf{x})\|_{F} d \mathbf{x}$. Considering two tensor fields $\mathbf{D}_{1}$ and $\mathbf{D}_{2}$, we show in the following that the gradient of our energy functional is $L$-Lipschitz. The constant $L$ will allow us to choose automatically a time step that insures the convergence of the algorithm.

$$
\begin{aligned}
\left\|\frac{\partial E_{\text {data }}}{\partial \mathbf{D}(\mathbf{x})}\left(\mathbf{D}_{1}\right)-\frac{\partial E_{\text {data }}}{\partial \mathbf{D}(\mathbf{x})}\left(\mathbf{D}_{2}\right)\right\|_{F} & =2 b^{2} \sum_{k=1}^{N}<\mathbf{G}_{k}, \mathbf{D}_{1}(\mathbf{x})-\mathbf{D}_{2}(\mathbf{x})>_{F} \\
& \leq 2 b^{2} \sum_{k=1}^{N}\left\|\mathbf{G}_{k}\right\|_{F}\left\|\mathbf{D}_{1}(\mathbf{x})-\mathbf{D}_{2}(\mathbf{x})\right\|_{F}
\end{aligned}
$$

Therefore $\left\|\nabla E_{\text {data }}\left(\mathbf{D}_{1}\right)-\nabla E_{\text {data }}\left(\mathbf{D}_{2}\right)\right\|_{T F} \leq 2 b^{2} \sum_{k=1}^{N}\left\|\mathbf{G}_{k}\right\|_{F}\left\|\mathbf{D}_{1}-\mathbf{D}_{2}\right\|_{T F}$. Besides, we can easily show the following inequality

$$
\left\|\nabla E_{\text {smooth }}\left(\mathbf{D}_{1}\right)-\nabla E_{\text {smooth }}\left(\mathbf{D}_{2}\right)\right\|_{T F} \leq 2\left(1+2\left|\mathcal{N}_{\mathbf{x}}\right|+\left|\mathcal{N}_{\mathbf{x}}\right|^{2}\right)\left\|\mathbf{D}_{1}-\mathbf{D}_{2}\right\|_{T F}
$$

where $\left|\mathcal{N}_{\mathbf{x}}\right|$ is the number of the considered neighbors. Thus the gradient of the objective function is $L$-Lipschitz with $L=2 b^{2} \sum_{k=1}^{N}\left\|\mathbf{G}_{k}\right\|_{F}+2 \lambda\left(\left|\mathcal{N}_{\mathbf{x}}\right|+\right.$ $1)^{2}$. Choosing $0<d t<\frac{1}{b^{2} \sum_{k=1}^{N}|| \mathbf{G}_{k}\|\|_{F}+\lambda\left(\left|\mathcal{N}_{\mathbf{x}}\right|+1\right)^{2}}$ makes the projected gradient descent convergent [14].

We can give an interpretation of our regularization energy in terms of diffusionweighted images smoothing. It can be easily verified that for each direction $k$

$$
\begin{array}{r}
\int_{\Omega}<\mathbf{D}(\mathbf{x})-\int_{\mathbf{y} \in \mathcal{N}_{\mathbf{x}}} \frac{w(\mathbf{x}, \mathbf{y})}{Z(\mathbf{x})} \mathbf{D}(\mathbf{y}) d \mathbf{y}, \mathbf{G}_{k}>_{F}^{2} d \mathbf{x}= \\
\frac{1}{b^{2}} \int_{\Omega}\left[\log \left(\frac{S_{k}(\mathbf{x})}{S_{0}(\mathbf{x})}\right)-\log \left(\prod_{\mathbf{y} \in \mathcal{N}_{\mathbf{x}}}\left(\frac{S_{k}(\mathbf{y})}{S_{0}(\mathbf{y})}\right)^{\frac{w(\mathbf{x}, \mathbf{y})}{Z(\mathbf{x})}}\right)\right]^{2} d \mathbf{x}
\end{array}
$$

Using Cauchy-Schwartz inequality we obtain :

$$
\frac{1}{b^{2}} \int_{\Omega}\left[\log \left(\frac{S_{k}(\mathbf{x})}{S_{0}(\mathbf{x})}\right)-\log \left(\prod_{\mathbf{y} \in \mathcal{N}_{\mathbf{x}}}\left(\frac{S_{k}(\mathbf{y})}{S_{0}(\mathbf{y})}\right)^{\frac{w(\mathbf{x}, \mathbf{y})}{Z(\mathbf{x})}}\right)\right]^{2} d \mathbf{x} \leq E_{\text {smooth }}\left\|\mathbf{G}_{k}\right\|_{F}^{2}
$$

We can see that minimizing $E_{\text {smooth }}$ has a direct implication on the normalized diffusion weighted images $\frac{S_{k}}{S_{0}}$. Reconstructing the tensors using a linear combination of the tensors in its neighborhood leads to the reconstruction of the normalized signals using a weighted geometric mean of the neighboring signals where the weights are not calculated only with a single volume $S_{k}$ but also with the volumes obtained from the other magnetic gradient directions. 


\section{Experimental Validation}

In order to validate the performance of the method we (i) have generated artificial tensors volumes corrupted with synthetic noise, (ii) used manual segmentation on T1 muscle images and tried to improve the separability of classes in the DTI space after regularization.

\subsection{Artificially Corrupted Tensors}

Let us consider two volumes, one that consists of two classes with orthogonal axes on a $20 \times 20 \times 20$ lattice and a helix in which the internal voxels are anisotropic and the external ones are spheric [Fig.1-b]. For the first volume, the tensor fields for each region are $\mathbf{D}_{\mathbf{1}}=0.001 \times\left[\begin{array}{llllll}1 & 0.5 & 0.5 & 0 & 0 & 0\end{array}\right]$ and $\mathbf{D}_{\mathbf{2}}=0.001 \times\left[\begin{array}{llllll}0.2 & 0.4 & 0.2 & 0 & 0 & 0\end{array}\right]$

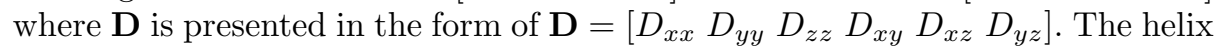
dataset can be found at [15]. We considered for both datasets a field strength $b=700 \mathrm{~s} . \mathrm{mm}^{-2}$, a constant value for $S_{0}=60$ for all volume voxels and twelve directions for diffusion gradient, which are used to generate the DTI corresponding to such tensor estimations. The chosen directions are the following :

$$
\left(\begin{array}{cccccccccccc}
1 & 1 & 1 & 1 & 0.41 & 0.41 & 0.41 & 0.41 & 0.41 & 0.41 & 0.41 & 0.41 \\
0.41 & -0.41 & -0.41 & 0.41 & 0.41 & 1 & 1 & 0.41 & -0.41 & -1 & -1 & -0.41 \\
-0.41 & -0.41 & 0.41 & 0.41 & 1 & 0.41 & -0.41 & -1 & -1 & -0.41 & 0.41 & 1
\end{array}\right)
$$

The images were corrupted with a white zero-mean Gaussian noise forming a data set where ground-truth on the tensor are available. An estimation of the tensor field relative to the noisy images provides the noisy tensors data.

Then, to perform comparisons we considered the regularization algorithm on noisy tensors presented in [6]. The following parameters were used for our method: $\lambda=50, \mathcal{N}_{\mathbf{x}}=3 \times 3 \times 3, d t=10^{-7}$ with 50 iterations. To evaluate the performance of these methods, we considered the average sum of squared differences (SSD) between the regularized tensors and ground truth ones. In [Table 1], we can see that our estimation and regularization approach achieves better results and produces a tensor close to the ground truth. Our method outperforms the one of [6] when the level of noise is relatively important. In fact, our method considers a more robust resemblance degree between voxels. Such a criterion insures a better selection of neighboring tensors involved in the estimation of a given tensor. On the other hand, the anisotropic diffusion based regularization relies on gradient information which is not robust in case of high noise. In order to assess qualitatively our algorithm, we reported in [Fig. 1] the resulting tensors using our regularization method and the constrained anisotropic one. We can observe that our method achieves a better direction preservation, even in the presence of a strong noise.

\subsection{DTI towards Understanding the Human Skeletal Muscle}

In order to perform validation using real data, the following experiment was considered. DTI acquisitions of human skeletal muscle (calf) using 12 directions were 


\begin{tabular}{|l|l|l|l|l|l|l|}
\hline & \multicolumn{3}{|c|}{ Helix dataset } & \multicolumn{3}{c|}{ Homogeneous regions } \\
\hline$\sigma_{n}$ & 0.5 & 1.2 & 3 & 1.5 & 4 & 9 \\
\hline Noisy Tensor & 1.08 & 6.24 & 39.54 & 9.82 & 71.25 & 393.38 \\
Method in [6] & 0.33 & 1.60 & 10.57 & 3.32 & 22.47 & 120.70 \\
\hline Our Method & 0.41 & 1.38 & 3.78 & 0.44 & 4.23 & 18.30 \\
\hline
\end{tabular}

Table 1. Average Sum of Square Differences (SSD) $\times 10^{4}$. Comparisons between our method and the one in [6]

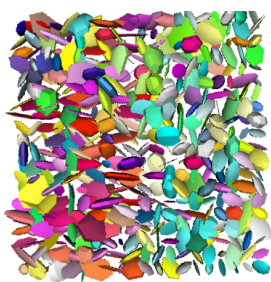

(a)

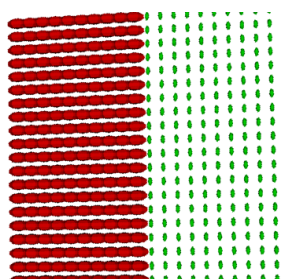

(b)

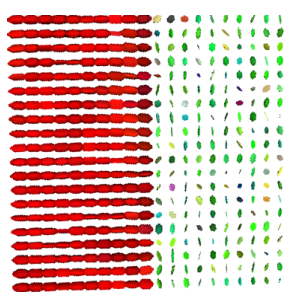

(c)

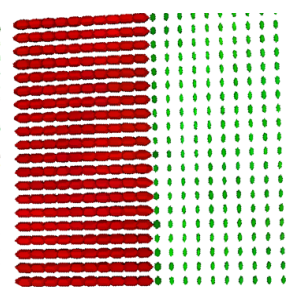

(d)

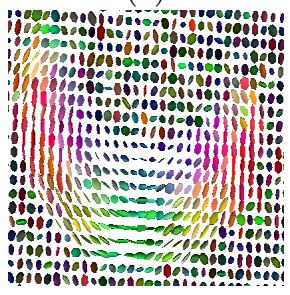

(a)

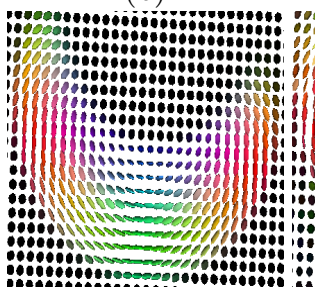

(b)

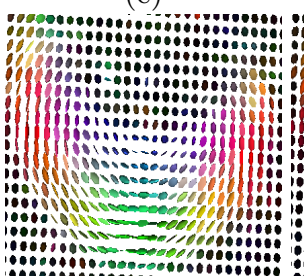

(c)

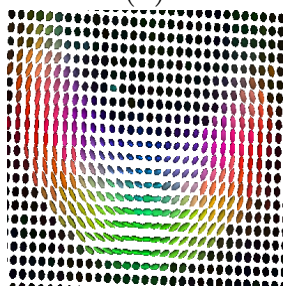

(d)

Fig. 1. Tensors on a volume slice: (a) Noisy tensors (b) Ground-truth (c) Result obtained with [6] (d) Result obtained with our method

carried out on a $1.5 \mathrm{~T}$ MRI scanner with the following parameters : repetition time $(\mathrm{TR})=3600 \mathrm{~ms}$, echo time $(\mathrm{TE})=70 \mathrm{~ms}$, slice thickness $=7 \mathrm{~mm}$ and $b$ value of $700 \mathrm{~s} . \mathrm{mm}^{-2}$. In order to improve the signal-to-noise ratio, the acquisition was repeated thirteen times (one can use the average of the measurements) while a high resolution T1-weighted volume was also obtained and manually segmented [Fig. 2]. The muscles that were considered in our study were the soleus (SOL), lateral gastrocnemius (LG), medial gastrocnemius (MG), posterior tibialis (PT), anterior tibialis (AT), extensor digitorum longus (EDL), and the peroneus longus (PL). Several previous studies investigated the use of diffusion tensor imaging to study the architecture of skeletal muscle and to separate these muscle groups according to different properties (fiber orientation, mean diffusivity, fractional anisotropy ...) $[16,17]$.

In order to proceed with an evaluation of the proposed method, the following scenario was considered: Using the manual segmentation, and the observed measurements of a given acquisition (12 directions), we have constructed seven weak linear classifiers (in our case a multi-class linear SVM[18]) separating each 
class of muscle versus all others. Then, the success rate (percentage of voxels being attributed to the right class) from the classifier with respect to the ground truth was determined. We remark that linear separation is hardly achieved for PT, PL, EDL and AT while it yields quite satisfactory results for the MG, LG and to a lesser extent SOL which form the major part of the muscle. We have performed this test thirteen times for: (i) direct estimation (DE), (ii) direct estimation and regularization (DER), as well as using direct estimation of the average measurements of the thirteen acquisitions (ADE). One would expect that since muscles consist of myo-fibers of the same nature, the classification should be improved if the estimation of the tensors is properly done, i.e. with appropriate regularization. However, it is important to note that the aim of this paper is not automatic classification of voxels in different muscle regions using DTI (in such a case more advanced classifiers can be used).
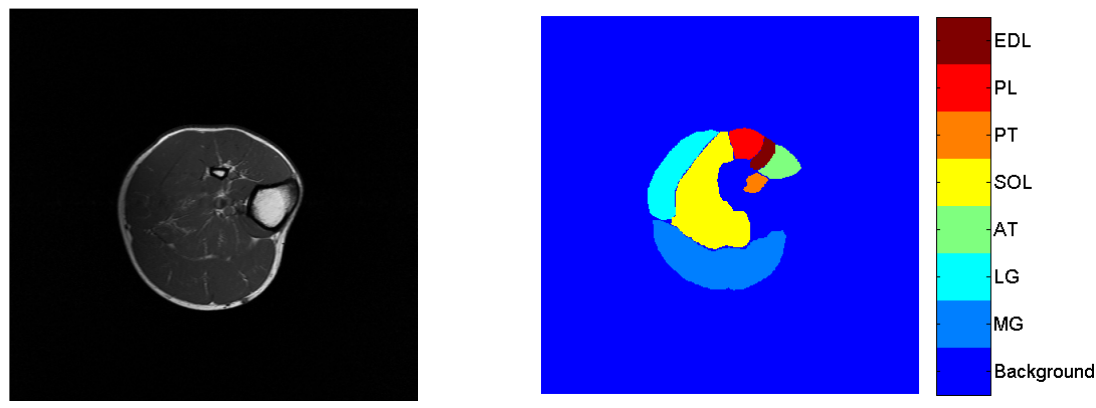

Fig. 2. A slice of the T1-weighted volume, different muscle groups segmented manually

In [Table 2], we present quantitative validation of the present framework for the linearly separable muscles. One can see that our method leads to an improvement in the correct classification rates with respect to a plain direct estimation. We also obtain better results when compared to the averaging+estimation method. We also show the result of our regularization on a slice of the volume in [Fig. 3].

\begin{tabular}{|c|c|c|c|c|}
\hline & Overall & MG & LG & SOL \\
\hline DE & $78.1 \%$ & $86.16 \%$ & $51.1 \%$ & $84.43 \%$ \\
\hline ADE & $84.46 \%$ & $90.47 \%$ & $65.72 \%$ & $88.43 \%$ \\
\hline DER & $86.45 \%$ & $91.82 \%$ & $69.76 \%$ & $89.97 \%$ \\
\hline
\end{tabular}

Table 2. Correct classification rates for the different methods and for each muscle group. The first and third row show the average correct classification rates for the set of 13 volumes 


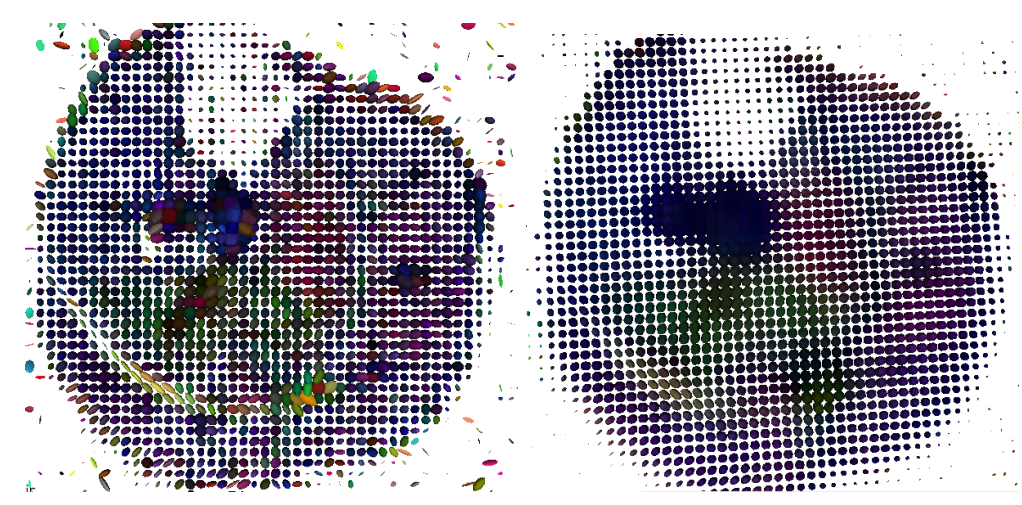

Fig. 3. Estimated tensors without regularization, tensors obtained with our method

\section{Discussion}

In this paper a novel approach to direct estimation and regularization of diffusion tensor images was proposed. The main strength of our approach is the novel regularization term that assumes linear approximation of neighborhood tensors as well as the convex nature of the proposed cost function which can be easily optimized. Our method was compared and outperformed the anisotropic constrained regularization using generated data with known noise model, and significantly improved human skeletal muscle segmentation/classification through DTI using real data.

The selection of the bandwidth $\sigma$ is a critical parameter of the process. Datadriven variable bandwidth models is a natural extension of the method. One would expect that the optimal bandwidth depends on the form of the observed anatomical structure which varies spatially. Another possible extension of this work is to replace the Frobenius norm in the energy functional by the Riemannian metric [5] or the Log-Euclidean metric [9]. However this will be done at the expense of the convexity of the function and the computational time.

The use of DTI towards understanding the human skeletal muscle as well as providing means of diagnosis for muscular diseases is a more long-term objective of our research. The ability to understand the remodeling of myofibers due to muscular diseases using non-invasive means is a great perspective.

Acknowledgements We are thankful to D. Tschumperlé for providing us with the implementation of the regularization algorithm [6] for comparison and also to J.-F. Deux, G. Bassez and A. Rahmouni from Henri Mondor Hospital for providing the DTI data.

\section{References}

1. Bihan, D.L., Mangin, J.F., Poupon, C., Clark, C.A., Pappata, S., Molko, N., Chabriat, H.: Diffusion tensor imaging: concepts and applications. Journal of 
Magnetic Resonance Imaging 13 (2001) 534-546

2. Coulon, O., Alexander, D.C., Arridge, S.: Diffusion tensor magnetic resonance image regularization. Medical Image Analysis 8 (2004) 47-67

3. Basu, S., Fletcher, P.T., Whitaker, R.T.: Rician noise removal in diffusion tensor mri. In: MICCAI (1). (2006) 117-125

4. Martín-Fernández, M., Westin, C.F., Alberola-López, C.: 3D Bayesian regularization of diffusion tensor MRI using multivariate Gaussian Markov random fields. In: MICCAI (1). (2004) 351-359

5. Castano-Moraga, C.A., Lenglet, C., Deriche, R., Ruiz-Alzola, J.: A Riemannian approach to anisotropic filtering of tensor fields. Signal Processing [Special Issue on Tensor Signal Processing], 87 (2007) 263-276

6. Deriche, R., Tschumperle, D., Lenglet, C., Rousson, M.: Variational approaches to the estimation, regularization and segmentation of diffusion tensor images. In Paragios, Chen, F., ed.: Mathematical Models in Computer Vision: The Handbook. 2005 edn. Springer (2005)

7. Wang, Z., Vemuri, B.C., Chen, Y., Mareci, T.H.: A constrained variational principle for direct estimation and smoothing of the diffusion tensor field from complex DWI. IEEE Transactions on Medical Imaging 23 (2004) 930-939

8. Weickert, J., Feddern, C., Welk, M., Burgeth, B., Brox, T.: PDEs for tensor image processing. In Weickert, J., Hagen, H., eds.: Visualization and Processing of Tensor Fields. Springer (2006) 399-414

9. Fillard, P., Arsigny, V., Pennec, X., Ayache, N.: Clinical DT-MRI estimation, smoothing and fiber tracking with log-Euclidean metrics. In: Proceedings of the IEEE International Symposium on Biomedical Imaging (ISBI 2006), Crystal Gateway Marriott, Arlington, Virginia, USA (2006) 786-789

10. Stejskal, E., Tanner, J.: Spin diffusion measurements: spin echoes in the presence of a time-dependent field gradient. Journal of Chemical Physics 42 (1965) 288-292

11. Salvador, R., Pea, A., Menon, D.K., Carpenter, T.A., Pickard, J.D., Bullmore, E.T.: Formal characterization and extension of the linearized diffusion tensor model. Human Brain Mapping 24 (2005) 144-155

12. Azzabou, N., Paragios, N., Guichard, F., Cao, F.: Variable bandwidth image denoising using image-based noise models. In: CVPR. (2007)

13. Hiriart-Urruty, J.B., Lemaréchal, C.: Fundamentals of Convex Analysis. Springer Verlag, Heidelberg (2001)

14. Bertsekas, D.P.: Nonlinear Programming. Athena Scientific, Belmont, MA (1999)

15. (www.sci.utah.edu/ ${ }^{\text {gk} / D T I-d a t a /) ~}$

16. Galban, C.J., Maderwald, S., Uffmann, K., de Greiff, A., Ladd, M.E.: Diffusive sensitivity to muscle architecture: a magnetic resonance diffusion tensor imaging study of the human calf. European Journal of Applied Physiology 93 (Dec 2004) $253-262$

17. Galban, C.J., Maderwald, S., Uffmann, K., Ladd, M.E.: A diffusion tensor imaging analysis of gender differences in water diffusivity within human skeletal muscle. NMR in Biomedicine (2005)

18. Joachims, T.: Making large-scale support vector machine learning practical. In B. Schölkopf, C. Burges, A.S., ed.: Advances in Kernel Methods: Support Vector Machines. MIT Press, Cambridge, MA (1998) 